\title{
WILEY-VCH
}

\section{Silver Oxalate: Mechanical Properties and Extreme Negative Mechanical Phenomena}

\section{Francisco Colmenero*}

Dr. Francisco Colmenero

Departamento de Física Molecular

Instituto de Estructura de la Materia (IEM-CSIC)

28006 Madrid, Spain

E-mail: francisco.colmenero@iem.cfmac.csic.es

Keywords: silver oxalate, mechanical properties, negative Poisson's Ratio, negative linear compressibility, density functional theory

\section{ABSTRACT}

The crystal structure and mechanical properties of silver oxalate, $\operatorname{Ag}_{2} \mathrm{C}_{2} \mathrm{O}_{4}$, are determined using first-principles solid-state methods. The computed structure and associated X-ray powder pattern are in excellent agreement with their experimental counterparts. The set of calculated properties include the bulk modulus and its pressure derivatives, the Young and shear moduli, the Poisson's Ratio and the ductility, hardness and anisotropy indices. Silver oxalate is a highly anisotropic brittle material possessing a small bulk modulus of $\sim 9.6 \mathrm{GPa}$. It displays the negative Poisson's ratio (NPR) phenomenon, the value of the lowest NPR having a very large magnitude, -1.27 . Besides, it exhibits the most extreme form of the negative linear compressibility (NLC) phenomenon found up to date. Silver oxalate displays anisotropic NLC for external pressures in the range -0.1 to $-2.4 \mathrm{GPa}$ directed along the [010] crystallographic direction and isotropic NLC for isotropic pressures in the range -0.51 to 13.4 $\mathrm{GPa}$. The lowest value of the negative compressibility, $-831.9 \pm 10 \mathrm{TPa}^{-1}$, is found for an isotropic pressure of $-0.16 \mathrm{GPa}$. The absolute value of the computed lowest NLC is extremely large, about three times larger than the absolute value of the lowest NLC found so far. The NLC pressure range is also very wide, its width being more than two times the largest range found up to date. 


\section{WILEY-VCH}

\section{MAIN TEXT.}

The mechanical properties of some small organic acids and metal-organic materials in the solid-state were obtained in previous works ${ }^{[1-6]}$ using theoretical solid-state methods based in Density Functional Theory (DFT) using plane waves and pseudopotentials. High-quality computational setups, comprising large plane wave basis sets and dense k-point meshes, were employed in order to obtain realistic descriptions of their mechanical behavior. These materials were shown to display negative Poisson's ratios (NPR) ${ }^{[7-13]}$ and to exhibit the important phenomenon of negative linear compressibility (NLC). ${ }^{[14-19]}$ The materials showing these phenomena have found an enormous range of applications because they have improved properties resulting specifically from their mechanical behavior. ${ }^{[1]}$ These properties make them appropriate in many applications as those involved in textile ${ }^{[20-24]}$ and automotive industries, ${ }^{[25]}$ personnel protection, ${ }^{[26]}$ biomedicine, ${ }^{[27-28]}$ aerospace and defense ${ }^{[29-32]}$ and in many commercial applications requiring filters, sieves, sensors, packaging and insulation. ${ }^{[16,33-34]}$ Negative linear compressibility, in particular, is a very relevant mechanical property which has been used for the development of ultrasensitive pressure-sensing devices, pressure driven actuators, optical telecommunication cables, artificial muscles, nextgeneration body armor and in biomedical uses. ${ }^{[1,16-17]}$ A broad review of the literature concerning the concepts, materials and applications related to these phenomena has been given in a previous paper. ${ }^{[1]}$ The materials exhibiting a negative value of the Poisson's ratio are often called auxetics ${ }^{[35]}$ and to distinguish them from materials that display negative Poisson's ratios only for certain directions of the applied strain, the term partial auxetics may be used. ${ }^{[36]}$

The number of known natural materials exhibiting NLC is very small. ${ }^{[19]}$ Synthetic and design approaches have been devised to obtain materials displaying specific mechanical properties. ${ }^{[1,18,37-39]}$ However, the magnitude of the negative compressibility and the range of applied pressures for which many of these natural and man-made materials show NLC is 


\section{WILEY-VCH}

frequently too small to be exploited in practical applications. For this reason, the search for

NLC materials has lost to some extent its initial impetus in recent years. The main motivation of this study and the previous ones, ${ }^{[1-6]}$ was to investigate the presence of negative mechanical phenomena in organic materials because these effects have rarely been studied in this kind of compounds, revealing a significant gap in their study. The interest comes from the large amount of potential applications of these materials, the great versatility of organic synthesis and the wide diversity of organic crystal structure types which may show these effects.

The lowest value of the NLC found so far is $-260 \mathrm{TPa}^{-1}$ for $\mathrm{CsH}_{2} \mathrm{PO}_{4},{ }^{[40-41]}$ the next lowest values being $-166 \mathrm{TPa}^{-1}$ for oxalic acid dihydrate ${ }^{[3]}$ and $-76 \mathrm{TPa}^{-1}$ for $\mathrm{Ag}_{3}\left[\mathrm{Co}(\mathrm{CN})_{6}\right] \cdot{ }^{[42-43]}$ These values were determined experimentally except that of oxalic acid dihydrate,${ }^{[3]}$ which was obtained recently using the theoretical solid-state methodology. The widest range of $\mathrm{NLC}$ pressures has been found experimentally for $\mathrm{BiB}_{3} \mathrm{O}_{6}$, ranging from 0.0 to $5.0 \mathrm{GPa} \cdot{ }^{[44-45]}$ In this communication, our theoretical results show that silver oxalate, $\mathrm{Ag}_{2} \mathrm{C}_{2} \mathrm{O}_{4}$, a very simple metal-organic material, exhibits the most extreme form of the negative linear compressibility phenomenon found up to date. The absolute value of the computed lowest negative compressibility is found to be about three times larger than the absolute value of the lowest compressibility found so far and the NLC pressure range is also very wide, its width being more than two times the largest range found up to date.

Anhydrous metal oxalates ${ }^{[46]}$ constitute an important group of materials having significant applications in nanomaterials technology ${ }^{[47-52]}$ because their controlled thermal decomposition process $^{[53-59]}$ lead to metallic or metal oxide products in the form of nanoparticles from which nanopowders possessing technologically desirable properties may be obtained. In particular, silver oxalate is a highly demanded material due to its use as a precursor for the production of silver nanoparticles which are incorporated in coatings, nanofibers, thin films, pigments, first aid bandages plastics, soaps, skin care products and textiles. ${ }^{[47,60-61]}$ Metallic ions as zinc and silver, have antimicrobial effects and therefore they are added at the stage of synthesis to a 


\section{WILEY-VCH}

large number of compounds used as dental composites and in artificial bone materials. ${ }^{[62]}$ Due to the importance of the thermal decomposition of silver oxalate, it has been studied using theoretical solid-state methods. ${ }^{[57-59]}$ Since, the decomposition of silver oxalate materials may also be activated mechanically ${ }^{[63-66]}$ it is surprising that the mechanical properties of this material have not been determined experimentally or theoretically and their anomalous mechanical behavior has not been noticed. As shown in this work, the mechanical behavior of silver oxalate is extremely interesting and their mechanical properties are potentially useful in a large series of applications.

The crystal structure of silver oxalate was studied using first-principles solid-state methods based in DFT using plane wave basis sets and pseudopotentials in order to describe the inner electrons of the atoms present in the unit cell of this material. ${ }^{[67]}$ The energy-density functional employed in this study was the specialized version of Perdew-Burke-Ernzerhof (PBE) functional for solid materials, PBEsol, ${ }^{[68]}$ as implemented in CASTEP code, ${ }^{[69]}$ a module of the Materials Studio package. ${ }^{[70]}$ The pseudopotentials used for C, O and Ag atoms in the unit cell of silver oxalate were standard norm-conserving pseudopotentials ${ }^{[71]}$ given in CASTEP code (00PBE-OP type). The atomic positions and unit cell lattice parameters were optimized using the Broyden-Fletcher-Goldfarb-Shanno method ${ }^{[72]}$ with a convergence threshold on atomic forces of $0.01 \mathrm{eV} / \AA$.

The calculation parameters, the plane wave kinetic energy cutoff and k-point mesh, employed in this computational study are given in Table S.1 of the Supporting Information. It must be emphasized that, as in the previous works, ${ }^{[1-6]}$ a very high quality computational setup was employed, much more accurate than those usually employed in this kind of studies. In the medium or fine standard quality degrees for a given calculation, the kinetic cutoff values are 650 and $765 \mathrm{eV}$, respectively, and a cutoff of $950 \mathrm{eV}$ was used in the present study. Likewise, the k-point mesh utilized in this work $(7 \times 4 \times 3)$ was constructed using a grid spacing of 0.04 $\AA$, and the medium and fine quality k-point meshes are constructed using grid spacings of 


\section{WILEY-VCH}

0.08 and $0.07 \AA$, respectively The calculated lattice parameters of the crystal structure of silver oxalate and the calculated atomic bond distances and angles are given in the Table S.2, Table S.3 and Table S.4 of the Supporting Information, respectively. In these Tables the calculated values are compared with the corresponding experimental data. ${ }^{[73]}$ As can be seen, the results are in excellent agreement with experiment, the differences in the computed unit cell volume and density being of the order of $1.1 \%$. Furthermore, the X-ray diffraction powder pattern of silver oxalate was calculated from the computed and experimental crystal structures. ${ }^{[74]}$ Figure 1 shows that the calculated pattern is in very good agreement with its experimental counterpart. The good agreement obtained for the crystal structure and X-ray diffraction pattern gives strong support to the theoretical treatment of silver oxalate used in this work.

The computed crystal structure of $\mathrm{Ag}_{2} \mathrm{C}_{2} \mathrm{O}_{4}$ is shown in Figure 2. The unit cell of this material contains oxalate ions, $\mathrm{C}_{2} \mathrm{O}_{2}^{2-}$, whose structure is only slightly distorted with respect to its structure in oxalic acid. ${ }^{[2]}$ The silver atoms in $\mathrm{Ag}_{2} \mathrm{C}_{2} \mathrm{O}_{4}$ form dimers in which each $\mathrm{Ag}$ atom displays a highly distorted tetrahedral coordination, $\mathrm{AgO}_{4}$. Two of the four coordinating oxygen atoms (denoted as $01^{\#}$ and $\mathrm{O2}^{\#}$ in Figure 2), belong to the same oxalate fragment and the other two belong to different second and third oxalate fragments.

The elasticity matrix needed to calculate the mechanical properties of $\mathrm{Ag}_{2} \mathrm{C}_{2} \mathrm{O}_{4}$ was obtained for the optimized crystal structure from stress-strain relationships employing the method of finite deformations. ${ }^{[75]}$ The derivatives of the bulk modulus with respect to pressure were determined by performing fits of the unit cell volumes and associated pressures to a fourth-order Birch-Murnahan ${ }^{[76]}$ equation of state (EOS). The unit cell volumes in the vicinity of the optimized structure were calculated by optimizing the geometry of $\operatorname{Ag}_{2} \mathrm{C}_{2} \mathrm{O}_{4}$ under the effect of eighteen different applied isotropic pressures within -1.0 to $11.0 \mathrm{GPa}$. The fits of the pressure-volume data to the chosen EOS were carried out using EOSFIT 5.2 computer 


\section{WILEY-VCH}

program. ${ }^{[77]}$ The calculated elasticity matrix, $C_{i j}$, is reported in Table S.5 of the Supporting Information. The satisfaction of the Born mechanical stability conditions ${ }^{[78-79]}$ was studied from the calculated elasticity matrix. For monoclinic crystals, the generic necessary and sufficient Born criterion is that all eigenvalues of the $C$ matrix be positive. ${ }^{[79]}$ The $C$ matrix was diagonalized numerically and all eigenvalues were found to be positive. Since this condition was fulfilled, it follows that the crystal structure of $\mathrm{Ag}_{2} \mathrm{C}_{2} \mathrm{O}_{4}$ is mechanically stable.

The mechanical properties of polycrystalline silver oxalate were determined using the Voigt, ${ }^{[80]}$ Reuss, ${ }^{[81]}$ and Hill ${ }^{[82]}$ schemes. As in previous studies, ${ }^{[1-2,5-6,83-86]}$ the Reuss approximation was chosen as the best approach in the case of silver oxalate because it provided the best comparison of the results for the calculated bulk modulus, $B=9.64 \pm 2.51$ GPa, with that determined from the EOS. The results are reported in Table 1. As may be seen, the calculated ductility index is $D=1.73$, and, consequently, silver oxalate is a brittle material. ${ }^{[87-88]}$ The calculated Vickers hardness, $H=0.85$, corresponds to a material of low hardness. ${ }^{[89]}$ The most important result of those given in Table 1, is the value of the universal anisotropy index ${ }^{[90]} A^{U}=26.64$. Since this value is very large, silver oxalate is a highly anisotropic material. The mechanical anisotropy results from the very different values of the elasticity matrix elements along the different directions, $C_{11}=44.09 \mathrm{GPa}, C_{22}=166.44 \mathrm{GPa}$ and $C_{33}=95.84 \mathrm{GPa}$ (see Table $\mathrm{S} .5$ of the Supporting Information). The large differences between these values is, in turn, due to the large differences in the bonding strength along the different directions in silver oxalate (see Figure 2).

Because there is a well-known symmetry independent correlation between the value of the mechanical anisotropy index and the minimal and maximal values of the Poisson's ratio, ${ }^{\text {[91-92] }}$ there were strong reasons to study this material in search for an anomalous mechanical behavior. Therefore, the dependence of the mechanical properties of $\mathrm{Ag}_{2} \mathrm{C}_{2} \mathrm{O}_{4}$ as functions of the direction of the applied strain was computed. Three-dimensional representations of the 


\section{WILEY-VCH}

mechanical properties of $\mathrm{Ag}_{2} \mathrm{C}_{2} \mathrm{O}_{4}$ as a function of the orientation of the applied strain were obtained using the ElAM software ${ }^{[92]}$ and are shown in Figure 3. As may be noticed in Figure 3.E and Table S.6 of the Supporting Information, silver oxalate displays a large negative value of the minimum Poisson's ratio, -1.27 , the longitudinal direction of minimum Poisson's ratio being $U_{\text {min }}^{L}=(-0.59,0.780 .20)$.

The results obtained in previous works, ${ }^{[1-6]}$ show that the presence of large negative Poisson's ratios is frequently accompanied by the presence of the important negative linear compressibility phenomenon. The compressibility is a mechanical property which measures the relative volume change of a material as a response to a pressure change. The compressibility must be strictly positive for space unconstrained solid materials which are thermodynamically and mechanically stable. ${ }^{[1,11,19,93-94]}$ Counterexamples to the general statement of positivity of the compressibility have been found for constrained systems. ${ }^{[93-94]}$ The volume of a material submitted to a hydrostatic (isotropic) pressure commonly does not increase, unless it is unstable. Negative linear compressibility (NLC) is not related to unstable materials and may refer ${ }^{[1-6,19]}$ to: (a) Anisotropic negative linear compressibility (ANLC): the positive variation of the volume when the material is submitted to a uniform compression directed along a given direction; and (b) Isotropic negative linear compressibility (INLC): the increase of one or two single directions of the unit cell of a material under the application of an isotropic pressure. In the first case the directional derivative of the volume with respect to pressure is negative. In the second case, the volume does not increase and the positive variation of the parameters in some directions is compensated by a larger negative variation of the parameters in the remaining directions. Clearly, the compressibility is, as the bulk, shear or Young moduli, response properties characterizing the behavior of a material submitted to external pressures and must be distinguished from intrinsic material properties as the total energy. 


\section{WILEY-VCH}

\section{Anisotropic negative linear compressibility (ANLC)}

Table S.7 of the Supporting Information, reports the optimized unit cell volumes and lattice parameters of silver oxalate for twenty different applied external pressures directed along the direction of minimum compressibility, $U_{\min }^{C}=(0.0,1.0,0.0)$ (the direction of $b$ axis or [010] crystallographic direction). The results are also plotted in Figure 4. As may be observed, the unit cell volume decreases upon application of positive pressures (compressive). However, it increases normally upon application of negative pressures up to about $-0.06 \mathrm{GPa}$ and then decreases. Therefore, silver oxalate displays NLC behavior upon application of negative pressures directed along $b$ axis. The range in which silver oxalate exhibits ANLC is quite large, from $-0.06 \mathrm{GPa}$ to at least $-2.0 \mathrm{GPa}$, the last value of the pressure considered. The pressure at which the linear compressibility becomes positive was determined from additional higher negative pressure calculations to be $-2.41 \mathrm{GPa}$. The computed compressibilities $\left(k_{V}=-1 / V \cdot(\partial V / \partial P)_{P}\right)$ in the range of pressures from 0.0 to $-1.9 \mathrm{GPa}$ are shown in Figure 5 and given in Table S.8 of the Supporting Information. The maximum value of the negative compressively, $k_{V}=-180.9 \pm 20 \mathrm{TPa}^{-1}$, is found for an applied pressure of $-0.18 \mathrm{GPa}$. The error estimate in the computed compressibility is an aggregate value of the possible errors due to the possible variations in the optimized crystal structures under pressure and in the numerical determination of the volume derivatives. The estimation of the variations in the computed compressibility with respect to possible modifications of the crystal structures was obtained from a study of the convergence of the unit cell volume with respect to the parameters employed in the computational treatment of this material, that is, from calculations of the compressibilities using increasingly more demanding calculation parameters. The absolute value of the anisotropic negative linear compressibility in silver oxalate is very large but smaller than the absolute value of the lowest negative linear 


\section{WILEY-VCH}

compressibility found up to date, $-260 \mathrm{TPa}^{-1} \cdot{ }^{[40-41]}$ However, as it will be shown below, the isotropic NLC effect in silver oxalate is much larger.

\section{Isotropic negative linear compressibility (INLC)}

The optimized unit cell volumes and lattice parameters of silver oxalate for twenty seven different applied external isotropic pressures are given in the Table S.9 of the Supporting Information and displayed in Figure 6. As may be observed, the $b$ lattice parameter increases continuously under compression from $-0.51 \mathrm{GPa}$. Therefore, silver oxalate exhibits INLC for an extremely large range of isotropic pressures beginning at the negative pressure of -0.51 GPa to positive pressures larger than $11.0 \mathrm{GPa}$. The pressure at which the linear compressibility becomes positive was determined from additional calculations to be 13.38 $\mathrm{GPa}$. The positive variation of the $b$ lattice parameter is compensated by the negative variation of the $a$ and $c$ lattice parameters so that the unit cell volume decreases in the full range of pressures considered. The computed compressibilities $\left(k_{b}=-1 / b \cdot(\partial b / \partial P)_{P}\right)$ in the range of pressures from $-0.45 \mathrm{GPa}$ to $0.45 \mathrm{GPa}$ is shown in Figure 7 and collected in Table S.10 of the Supporting Information. The maximum value of the negative compressively, $k_{b}=-831.9 \pm 20 \mathrm{TPa}^{-1}$ is found at $-0.16 \mathrm{GPa}$. The absolute value of this negative linear compressibility is by far larger than the absolute value of the lowest negative linear compressibility found up to date, $-260 \mathrm{TPa}^{-1}$ for $\mathrm{CsH}_{2} \mathrm{PO}_{4},{ }^{[40-41]}$ and therefore also larger than the absolute value of -166 or $-76 \mathrm{TPa}^{-1}$ reported for oxalic acid dihydrate ${ }^{[3]}$ and $\mathrm{Ag}_{3}\left[\mathrm{Co}(\mathrm{CN})_{6}\right],{ }^{[42-43]}$ respectively, which are considered to be very large. The range of external pressures in which silver oxalate shows INLC is also the largest found so far. For example, the NLC pressure range found for $\mathrm{BiB}_{3} \mathrm{O}_{6}$, is from 0.0 to $5.0 \mathrm{GPa} \cdot{ }^{[44-45]}$ and that for MIL-53(Al) is from 0.0 to $3.0 \mathrm{GPa} .{ }^{[95]}$ 


\section{WILEY-VCH}

\section{Crystal structure deformation}

ANLC. The values of a set of selected interatomic distances and angles and the lattice parameters and unit cell volumes for three different applied pressures $(-0.331,-0.177$ and 0.0 GPa) directed along $U_{\text {min }}^{C}$ direction are given in Table S.11 of the Supplementary Information. The meaning of these distances and angles may be very easily understood by looking at Figure 2. From the $\mathrm{C}-\mathrm{C}, \mathrm{C}-\mathrm{O}, \mathrm{O}-\mathrm{C}-\mathrm{O}$ and $\mathrm{O}-\mathrm{C}-\mathrm{C}$ sections of the Table, it follows that the geometry of the oxalate ions in silver oxalate change only very slightly under the applied pressures. Similarly, from the $\mathrm{Ag}-\mathrm{O}$ and $\mathrm{O}-\mathrm{Ag}-\mathrm{O}$ sections, one may observe that the $\mathrm{AgO}_{4}$ tetrahedra in silver oxalate also change slightly under pressure. The main changes appearing under the application of negative (expansive) pressures are the decrease of the $\mathrm{Ag}-$ $\mathrm{Ag}^{*}$ distances (placed along the $a$ axis) and the increase of $\mathrm{Ag}-\mathrm{Ag}^{* *}$ distances (placed along the $b$ axis). These changes in the distances between the different $\mathrm{AgO}_{4}$ tetrahedra, placed in the same plane perpendicular to the $c$ axis, are directly responsible for the variations of the $a$ and $b$ lattice parameters because, as it can be noticed in Table S.11, the variation of these distances is identical to the variations in the values of the lattice parameters. The application of pressure leads also to a slight flattening of the $\mathrm{AgO}_{4}$ tetrahedra, because the angles $01-$ $\mathrm{Ag}-\mathrm{O} 2$ and $\mathrm{O1}^{\#}-\mathrm{Ag}-\mathrm{O}^{\#}$ increase under the negative pressures. In turn, this flattening leads to the decrease of the $c$ lattice parameter. Therefore, the ANLC in silver oxalate results from the changes on the relative distances between the $\mathrm{AgO}_{4}$ tetrahedra along the $a$ and $b$ directions and the flattening of these tetrahedra under the effect of expansive pressures. While the increase of the $b$ lattice parameter and the decrease of the parameters in the orthogonal directions is the expected behavior for a material submitted to an expansive pressure directed along the $b$ direction, the large decrease of the $a$ parameter and the smaller one of $c$ parameter are not compensated by the increase of $b$, and the total volume decreases. This decrease of the 


\section{WILEY-VCH}

unit cell volume under expansive pressures is the cause for the anisotropic NLC phenomenon in silver oxalate.

INLC. The values of the same set of interatomic distances and angles as in the previous paragraph for three different applied isotropic pressures $(-0.253,0.0$ and $0.241 \mathrm{GPa})$ are given in Table S.12 of the Supplementary Information. Again, from the C - C, C - O, O $\mathrm{C}-\mathrm{O}$ and $\mathrm{O}-\mathrm{C}-\mathrm{C}$ sections of the Table, it follows that the geometry of the oxalate ions in silver oxalate change only very slightly under the applied isotropic pressures, and the same occurs (see the $\mathrm{Ag}-\mathrm{O}$ and $\mathrm{O}-\mathrm{Ag}-\mathrm{O}$ sections of Table S.12) for the $\mathrm{AgO}_{4}$ tetrahedra. The main changes appearing under the application of positive (compressive) isotropic pressures are the diminution of the $\mathrm{Ag}-\mathrm{Ag}^{*}$ distances and the increase of $\mathrm{Ag}-\mathrm{Ag}^{* *}$ distances which, as mentioned in the previous paragraph, are directly responsible for the variations of the $a$ and $b$ lattice parameters, respectively. The application of pressure leads in this case to a slight narrowing of the $\mathrm{AgO}_{4}$ tetrahedra, because the angles $\mathrm{O} 1-\mathrm{Ag}-\mathrm{O} 2$ and $01^{\#}-\mathrm{Ag}-02^{\#}$ decrease under the positive pressures. The narrowing is, in this case, the main geometrical variation leading to the increase of the $c$ lattice parameter. The increase of $b$ lattice parameter is very large for silver oxalate submitted to compressive isotropic pressures, leading to the extremely large INLC observed in this material.

\section{Conclusions}

The crystal structure and mechanical properties of silver oxalate were determined using first principles solid-state methods. The description of solid-state crystalline compounds using computational modelling techniques, is sufficiently advanced nowadays to predict their mechanical properties in good agreement with experimental measurements. ${ }^{[96-121]}$ The present computations were carried out separately with several different sets of calculation parameters associated to increasingly more demanding computations and the analysis of the convergence of the calculated crystal structures, associated energies and properties showed that they were 


\section{WILEY-VCH}

nearly converged with respect to the calculation parameters. Thus, the present results must be reproducible if the calculations are performed with accurate enough computational methods and a sufficient level of complexity associated to the calculation parameters employed. Consequently, due to the relevance of the present results, an experimental validation of the theoretical results reported in this paper should be carried out. The present work establishes silver oxalate as the largest NLC material known so far, the magnitude of the lowest linear compressibility and the NLC range being about three times larger than any previously reported values.

An additional conclusion which may be extracted from this work is that we should try to avoid the use of terms as giant or colossal to refer to a large NLC effect. Since the difference with respect to the previous findings is very large, the possibility of discovering even larger NLC effects is indeed significant. Furthermore, this possibility should stimulate the investigation in this research field, particularly in what concerns to the systematic search of new NLC materials, mechanisms and applications.

The main changes observed in the crystal structure deformations associated to the application of both anisotropic and isotropic pressures have been seen to be the variations of the relative distances of the silver coordination polyhedra along the [100] and [010] crystallographic directions and the change in shape of these polyhedra. Since the silver tetrahedra are relatively distant to each other and bonded only in an indirect way, it may be concluded that the mechanism of NLC in this material is unrelated to the wine-rack structural model $^{[14,42-43,95,106-107,122-125]}$ frequently used to rationalize this phenomenon. Similarly, the mechanism appears to be different to the most important mechanisms found so far ${ }^{[19]}$ as the ferroelastic instabilities, ${ }^{[126-127]}$ phonon instabilities, ${ }^{[128-129]}$ reverse ferroelastic phase transitions ${ }^{[130-131]}$ correlated polyhedral tilts, ${ }^{[40-41,44-45,99,132-133]}$ helical structural motifs ${ }^{[120,134-}$ ${ }^{136]}$ and crystal structures involving chains or sheets held together by means of weak van der Waals interactions. ${ }^{[1-4]}$ 


\section{WILEY-VCH}

\section{Supporting Information}

Supporting Information is available from the Wiley Online Library or from the author.

\section{Acknowledgements}

Supercomputer time by the CTI-CSIC center is greatly acknowledged. I want to thank Prof.

Rafael Escribano for reading the manuscript and many helpful comments.

Received: ((will be filled in by the editorial staff))

Revised: ((will be filled in by the editorial staff)) Published online: ((will be filled in by the editorial staff))

\section{References}

[1] F. Colmenero, Mater. Res. Express 2019, 6, 045610.

[2] F. Colmenero, Phys. Chem. Chem. Phys. 2019, 21, 2673.

[3] F. Colmenero, Mater. Lett. 2019, 245, 25.

[4] F. Colmenero, Mater. Res. Express 2019, 6, 069401.

[5] F. Colmenero, Appl. Sci. 2018, 8, 2281.

[6] F. Colmenero, J. Cobos and V. Timón, J. Phys.: Condens. Matter 2019, 31, 175701.

[7] R. S. Lakes, Science 1987, 235, 1038.

[8] R. S. Lakes, Adv. Mater. 1993, 5, 293.

[9] J. N. Grima, R. Jackson, A. Alderson and K. E. Evans, Adv. Mater. 2000, 12, 1912.

[10] G. N. Greaves, R. S. Lakes and T. Rouxel, Nat. Mater. 2011, 10, 823.

[11] R. S. Lakes, Annu. Rev. Mater. Res. 2017, 47, 63.

[12] K.W. Wojciechowski, Mol. Phys. 1987, 61, 1247.

[13] K.W. Wojciechowski, Phys. Lett. A 1989, 137, 60.

[14] R. H. Baughman, S. Stafström, C. Cui and S. O. Dantas, Science 1988, 279, 1522. 


\section{WILEY-VCH}

[15] J. A. Kornblatt, E. B. Sirota, H. E. King, R. H. Baughman and C. Cui, Science 1988, 281, 143.

[16] K. E. Evans and A. Alderson, Adv. Mater. 2000, 12, 617.

[17] G. M. Spinks, G. G. Wallace, L. S. Fifield, L. R. Dalton, A. Mazzoldi, D. De Rossi, I. I. Khayrullin and R. H. Baughman, Adv. Mater. 2002, 14, 1728.

[18] C. N. Weng, K. T. Wang and T. Chen, Adv. Mater. Res. 2008, 33-37, 807.

[19] A. B. Cairns and A. L. Goodwin, Phys. Chem. Chem. Phys. 2015, 17, 20449.

[20] A. Alderson and K. L. Alderson, Tech. Text. Int. 2005, 14, 29.

[21] N. Ravirala, K. L. Alderson, P. J. Davies, V. R. Simkins and A. Alderson, Text. Res. J. 2006, 76, 540-546.

[22] S. C. Ugbolue, Y. K. Kim, S. B. Warner, Q. Fan, C. L. Yang. O. Kyzymchuk and Y. Feng, J. Text. Inst. 2010, 101, 660-667

[23] H. Hu, Z. Y. Wang, and S. Liu, Text. Res. J. 2011, 81, 1493-1502

[24] Z. Wang and H. Hu, Text. Res. J. 2014, 84, 1600.

[25] Z. D. Ma, Y. Y. Liu, X. M. Liu, C. Sun, Y. Cui Y, US Pat. 2011, 2011/0168313A1.

[26] F. Scarpa, J. Giacomin, Y. Zhang and P. Pastorino, Cell. Polym. 2005, 24, 253.

[27] W. Dolla, B. A. Fricke and B. R. Becker, J. Med. Devices 2007, 1, 47.

[28] F. Scarpa, IEEE Sign Proc. Mag. 2008, 25, 126.

[29] C. Lira, F. Scarpa, R. A. Rajasekaran, J. Intel. Mater. Syst. Str. 2011, 22, 907.

[30] Q, Liu, Report DSTO-GD-0472 (DSTO Formal Reports, Sydney, 2006).

[31] D. Bornengo, F. Scarpa and C. Remillat, J. Aerosp. Eng. 2005, 219, 185.

[32] J. Martin, J. Heyder-Bruckner, C. Remillat, F. Scarpa, K. Potter and M. Ruzzene, Phys. Stat. Sol. B 2008, 245, 570.

[33] A. Alderson, J. Rasburn, K. E. Evans, J. N. Grima, N, Membr. Technol. 2001, 137, 6.

[34] A. Alderson, J. Rasburn, S. Ameer-Beg, P. G. Mullarkey, W. Perrie and K. E. Evans, Ind. Eng. Chem. Res. 2000, 39, 654. 


\section{WILEY-VCH}

[35] K. E. Evans, Endeavour 1991, 15, 170.

[36] A. C. Branka, D. M. Heyes, K.W. Wojciechowski, Phys. Status Solidi B 2011, 248, 96.

[37] J. H. Lee, J. P. Singer and E. L. Thomas, Adv. Mater. 2012, 24, 4782.

[38] S. Babee, J. Shim, J. C. Weaver, E. R. Chen, N. Patel and K. Bertoldi, Adv. Mater. 2013, 25,5044 .

[39] J. N. Grima, S. Winczewski, L. Mizzi, M. C. Grech, R. Cauchi, R. Gatt, D. Attard, K. W. Wojciechowski and J. Rybicki, Adv. Mater. 2014, 27, 1455.

[40] S. Prawer, T. F. Smith and T. R. Finlayson, Aust. J. Phys. 1985, 38, 63.

[41] Y. Kobayashi, K. Deguchi, S. Azuma, E. Suzuki, L. C. Ming, S. Endo and T. Kikegawa, Ferroelectrics 2003, 285, 83.

[42] A. L. Goodwin, M. Calleja, M. J. Conterio, M. T. Dove, J. S. O. Evans, D. A. Keen, L. Peters and M. G. Tucker, Science 2008, 319, 794.

[43] A. L. Goodwin, D. A. Keen and M. G. Tucker, Proc. Natl. Acad. Sci. U. S. A. 2008, 105, 18708.

[44] S. Haussuhl, L. Bohaty and P. Becker, Appl. Phys. A 2006, 82, 495.

[45] R. Dinnebier, B. Hinrichsen, A. Lennie and M. Jansen, Acta Crystallogr. B 2009, 65, 1. [46] E. J. Baran, J. Coord. Chem. 2014, 67, 3734.

[47] S. Zhang, Y. Tang and B. Vlahovic, Nanoscale Res. Lett. 2016, 11, 80.

[48] T. Ahmad, A. Ganguly, J. Ahmed, A. K. Ganguli, O. Abdullah and A. Alhartomy, Arab. J. Chem. 2011, 4, 125.

[49] B. Sengupta, C. A. Tamboli and R. Sengupta, Chem. Eng. J. 2011, 169, 379.

[50] X. L. Zhou, Z. G. Yan and X. DongHan, Mater. Lett. 2014, 118, 39.

[51] K. Sarada, K.R. Vijisha and K. Muraleedharan, J. Anal. Appl. Pyrol. 2016, 120, 207.

[52] L. Tao, L. Ying and M. Guohua, Rare Metal Mater. Eng. 2017, 46, 2371.

[53] D. Dollimore, Thermochim. Acta 1987, 117, 331.

[54] D. Dollimore, Thermochim. Acta 1990, 177, 59. 


\section{WILEY-VCH}

[55] A. K. Galwey and M. E. Brown, J. Therm. Anal. Cal. 2007, 90, 9.

[56] B. Małecka, E. Drozdz-Ciesla, A. Małecki, Thermochim. Acta 2004, 423, 13.

[57] A. Kolezynski and A. Małecki, J. Thermal Anal. Cal. 2009, 96, 161

[58] A. Kolezynski and A. Małecki, J. Thermal Anal. Cal. 2009, 97, 167

[59] A. Kolezynski, Physica B 2010, 405, 3650.

[60] Y. Dong, X. Li, S. Liu, Q. Zhua, J. G. Li, and X. Sun, Thin Solid Films 2015, 589, 381.

[61] H. C. Lin, P. Lin, C. A. Lu, S. F. Wang, Microelectron. Eng. 2009, 862316.

[62] R. Stencel, J. Kasperski, W. Pakieła, A. Mertas, E. Bobela, I. Barszczewska-Rybarek, and G. Chladek, Materials 2018, 11, 1031.

[63] F. Delogu, Mater. Chem. Phys. 2012, 137, 297.

[64] F. Delogu, Mater. Chem. Phys. 2014, 147, 629.

[65] F. Delogu, Acta Mater. 2014, 66, 388.

[66] G. Ligios, A. M. Bertetto, F. Delogu, J. Alloys Compd. 2013, 554, 426.

[67] M. C. Payne, M. P. Teter, D. C. Ailan, A. Arias and J. D. Joannopoulos, Rev. Mod. Phys. 1992, 64, 1045.

[68] J. P. Perdew, A. Ruzsinszky, G. I. Csonka, O. A. Vydrov, G. E. Scuseria, L. A.

Constantin, X. Zhou and K. Burke, Phys. Rev. Lett. 2008, 100, 136406.

[69] S. J. Clark, M. D. Segall, C. J. Pickard, P. J. Hasnip, M. I. J. Probert, K. Refson and M.

C. Payne, Z. Kristallogr. 2005, 220, 567.

[70] MaterialsStudio, http://3dsbiovia.com/products/collaborative-science/biovia-materialsstudio/, accessed: December, 2018.

[71] N. Troullier, J. L. Martins, Phys. Rev. B. 1991, 43, 1993.

[72] B. G. Pfrommer, M. Cote, M. S. G. Louie, M. L. Cohen, J. Comp. Phys. 1997, 131.

[73] D.Y. Naumov, A.V. Virovets, N.V. Podberezkaya, E.V. Boldyreva, Acta Crystallogr. C 1995, 51, 60 . 


\section{WILEY-VCH}

[74] R. T. Downs, K. L. Bartelmehs, G. V. Gibbs and M. B. Boisen, Am. Mineral. 1993, 78, 1104.

[75] R. Yu, J. Zhu and H. Ye, Comput. Phys. Commun. 2010, 181, 671.

[76] F. Birch, Phys. Rev. 1947, 71, 809.

[77] R. J. Angel, Mineral. Geochem. 2000, 41, 35.

[78] M. Born, Math. Proc. Cambridge Phil. Soc. 1940, 36, 160.

[79] F. Mouhat and F. X. Coudert, Phys. Rev. B 2014, 90, 224104.

[80] W. Voigt, Lehrbuch der Kristallphysik, Teubner, Leipzig, 1962.

[81] A. Reuss, Z. Angew. Math. Mech., 1929, 9, 49.

[82] R. Hill, Proc. Phys. Soc. Lond. A 1952, 65, 349.

[83] F. Colmenero, L. J. Bonales, J. Cobos and V. Timón, J. Solid. State Chem. 2017, 253, 249.

[84] F. Colmenero, L. J. Bonales, J. Cobos and V. Timón, J. Phys. Chem. C 2017, 121, 5994.

[85] F. Colmenero, J. Cobos and V. Timón, Inorg. Chem. 2018, 57, 4470-4481.

[86] F. Colmenero, A. M. Fernández, J. Cobos and Timón, RSC Adv. 2018, 8, 24599.

[87] S. F. Pugh, Phil. Mag. 1954, 45, 823.

[88] Y. Bouhadda, S. Djella, M. Bououdina, Y. Fenineche and Y. Boudouma, J. Alloys Compd. 2012, 534, 20.

[89] H. Niu, P. Wei, Y. Sun, C. X. Chen, C. Franchini, D. Li and Y. Li Y, Appl. Phys. Lett. 2011, 99, 031901.

[90] S. I. Ranganathan and M. Ostoja-Starzewski, Phys. Rev. Lett. 2008, 101, 055504.

[91] Z. A. D. Lethbridge, R. I. Walton, A. S. H. Marmier, C. W. Smith and K. E. Evans, Acta Mater. 2010, 58, 6444.

[92] A. Marmier, Z. A. D. Lethbridge, R. I. Walton, C. W. Smith, S. C. Parker and K. E. Evans, Comput. Phys. Commun. 2010, 181, 2102.

[93] R. S. Lakes and K. W. Wojciechowski, Phys. Stat. Sol. (b) 2008, 245, 545. 


\section{WILEY-VCH}

[94] J. N. Grima, R. Caruana-Gauci, Nat. Mater. 2012, 11, 565.

[95] P. Serra-Crespo, A. Dikhtiarenko, E, Stavitski, J. Juan-Alcañiz, F. Kapteijn, F.-X.

Coudert and J. Gascon, CrystEngComm 2015, 17, 276.

[96] N. R. Keskar and J. R. Chelikowsky, Nature 1992, 358, 222.

[97] J. N. Grima, R. Gatt, A. Alderson and K. E. Evans, Mater. Sci. Eng. A 2006, 423, 219.

[98] J. N. Grima, R. Gatt, V. Zammit, J. J. Williams, K. E. Evans, A. Alderson, R. I. Walton, J. Appl. Phys. 2007, 101, 086102.

[99] F. X. Coudert, Phys. Chem. Chem. Phys. 2013, 15, 16012.

[100] F. X. Coudert, Chem. Mater. 2017, 29, 7833.

[101] Y. T. Yao, K. L. Alderson and A. Alderson, Cellulose 2016, 23, 3429.

[102] J. C. Tan, B. Civalleri, A. Erba, E. Albanese, CrystEngComm 2015, 17, 375.

[103] M. R. Ryder and J. C. Tan, Dalton Trans. 2016, 45, 4154.

[104] H. Sun, S. Mukherjee and C. V. Singh, Phys. Chem. Chem. Phys. 2016, 18, 26736.

[105] Y. Du, J. Maassen, W. Wu, Z. Luo, X. Xu, P. D. Ye, Nano Lett. 2016, 16, 6701.

[106] W. Li, M. R. Probert, M. Kosa, M. T. D. Bennett, A. Thirumurugan, R. P. Burwood, M.

Parinello, J. A. Howard, A. K. Cheetham, J. Am. Chem. Soc. 2012, 134, 11940.

[107] Y. Qiao, K. Wang, H. Yuan, K. Yang, B. Zou, J. Phys. Chem. Lett. 2015, 6, 2755.

[108] C. S. Coates, J. W. Makepeace, A. G. Seel, M. Baise, B. Slater, A. L. Goodwin, Dalton Trans. 2018, 47, 7263.

[109] A. Marmier, P. S. Ntoahae, P. E. Ngoepe, D. G. Pettifor, S. C. Parker, Phys. Rev. B 2010, $81,172102$.

[110] L. Kang, X. Jiang, S. Luo, P. Gong, W. Li, X. Wu, Y. Li, X. Li, C. Chen, Z. Lin, Sci. Rep. 2016, 5, 13432.

[111] J. Dagdelen, J. Montoya, M. De Jong, K. Persson, Nat. Commun. 2017, 8, 323.

[112] D. T. Ho, S. D. Park, S. Y. Kwon, K. Park, S. Y. Kim, Nat. Commun. 2014, 5, 3255. 


\section{WILEY-VCH}

[113] D. Wu, S. Wang, S. Zhan, S. J. Yuan, B. Yang, H. Chen, Phys. Chem. Chem. Phys. 2018, 20, 18924.

[114] F. Hao, X. Liao, M. Li, H. Xiao, X. Chen, J. Phys.: Cond. Matter 2018, 30, 315302.

[115] H. Wang, Q. Li, Y. Gao, F. Miao, X. Zhou, X. G. Wan, New J. Phys. 2016, 18, 073016.

[116] H. Wang, Z. D. Zhang, R. Q. Wu, L. Z. Sun, Acta Mater. 2013, 61, 2919.

[117] H. Wang, X. Li, P. Li, J. Yang, Nanoscale 2017, 9, 850.

[118] X. F. Wang, T. E. Jones, W. Li, Y. C. Zhou, Phys. Rev. B 2012, 85, 134108.

[119] S. Yalameha, A. Vaez, Int. J. Mod. Phys. B 2018, 32, 1850129.

[120] H. Hsueh, C. C. Lee, C. W. Wang, Phys. Rev. B 2000, 61, 3851.

[121] W. Ren, J. Ye, W. Shi, Z. K. Tang, C. T. Chan, P. Sheng, New J. Phys. 2009, 11, 103014.

[122] A. B. Cairns, A. L. Thompson, M. G. Tucker, J. Haines and A. L. Goodwin, J. Am. Chem. Soc. 2012, 134, 4454.

[123] C. H. Woodall, C. M. Beavers, J. Christensen, L. E. Hatcher, M. Intissar, A. Parlett, S.

J. Teat, C. Reber and P. R. Raithby, Angew. Chem., Int. Ed. 2013, 52, 9691.

[124] A. D. Fortes, E. Suard and K. S. Knight, Science 2011, 331, 742.

[125] M. R. Ryder, M. R. Ciballeri and J.-C. Tan, Phys. Chem. Chem. Phys. 2016, 18, 9079.

[126] T. G. Worlton and R. A. Beyerlein, Phys. Rev. B 1975, 12, 1899.

[127] P. S. Peercy, I. J. Fritz and G. A. Samara, J. Phys. Chem. Solids 1975, 36, 1105.

[128] I. E. Collings, A. B. Cairns, A. L. Thompson, J. E. Parker, C. C. Tang, M. G. Tucker, J.

Catafesta, C. Levelut, J. Haines, V. Dmitriev, P. Pattison and A. L. Goodwin, J. Am. Chem. Soc. 2013, 136, 7610.

[129] S. H. Lapidus, G. J. Halder, P. J. Chupas and K. W. Chapman, J. Am. Chem. Soc. 2013, $135,7621$.

[130] D. L. Decker, S. Petersen, D. Debray and M. Lambert, Phys. Rev. B 1979, 19, 3552.

[131] R. J. Angel, U. Bismayer and W. G. Marshall, Acta Crystallogr. B 2004, 60, 1. 


\section{WILEY-VCH}

[132] K. de Boer, A. Jansen and R. van Santen, Phys. Rev. B 1996, 52, 12579.

[133] J. Haines, C. Chateau, J. M. Leger, C. Bogicevic, S. Hull, D. D. Klug and J. S. Tse, Phys. Rev. Lett. 2003, 91, 015503.

[134] D. R. McCann, L. Cartz, R. E. Schmunk and Y. D. Harker, J. Appl. Phys. 1972, 43, 1432.

[135] P. W. Bridgman, Proc. Am. Acad. Arts Sci. 1945, 76, 9.

[136] A. B. Cairns, J. Catafesta, C. Levelut, J. Rouquette, A. van der Lee, L. Peters, A. L. Thompson, V. Dmitriev, J. Haines and A. L. Goodwin, Nat. Mater. 2013, 12, 212. 


\section{WILEY-VCH}

Figures and Tables

Figure 1. X-ray powder patterns of silver oxalate determined from the calculated and experimental ${ }^{[73]}$ geometries using $\mathrm{CuK}_{\alpha}$ radiation.

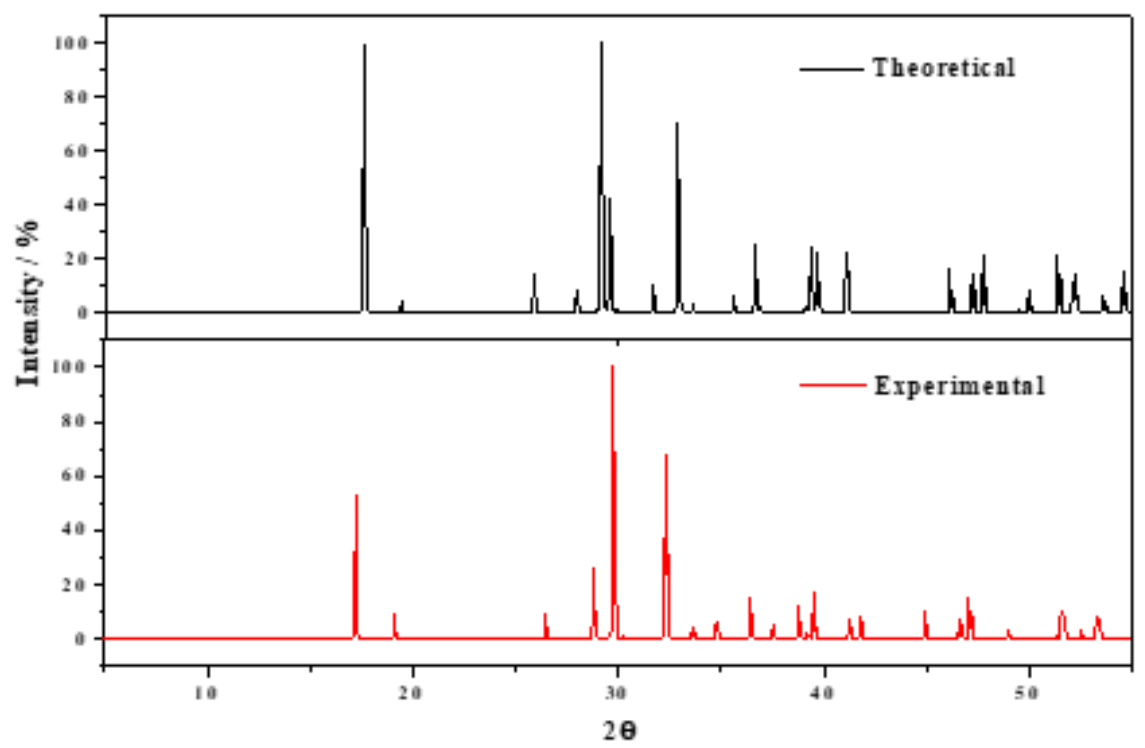

Figure 2. Computed crystal structure of silver oxalate: view of a $2 \times 2 \times 2$ supercell from [100] direction. Color code: Ag-blue; O-red; C-grey. The labelling convention used for the atoms in $\mathrm{Ag}_{2} \mathrm{C}_{2} \mathrm{O}_{4}$ and the $\mathrm{Ag}-\mathrm{Ag}^{*}$ and $\mathrm{Ag}-\mathrm{Ag}^{* *}$ interatomic distances are also shown in the Figure.

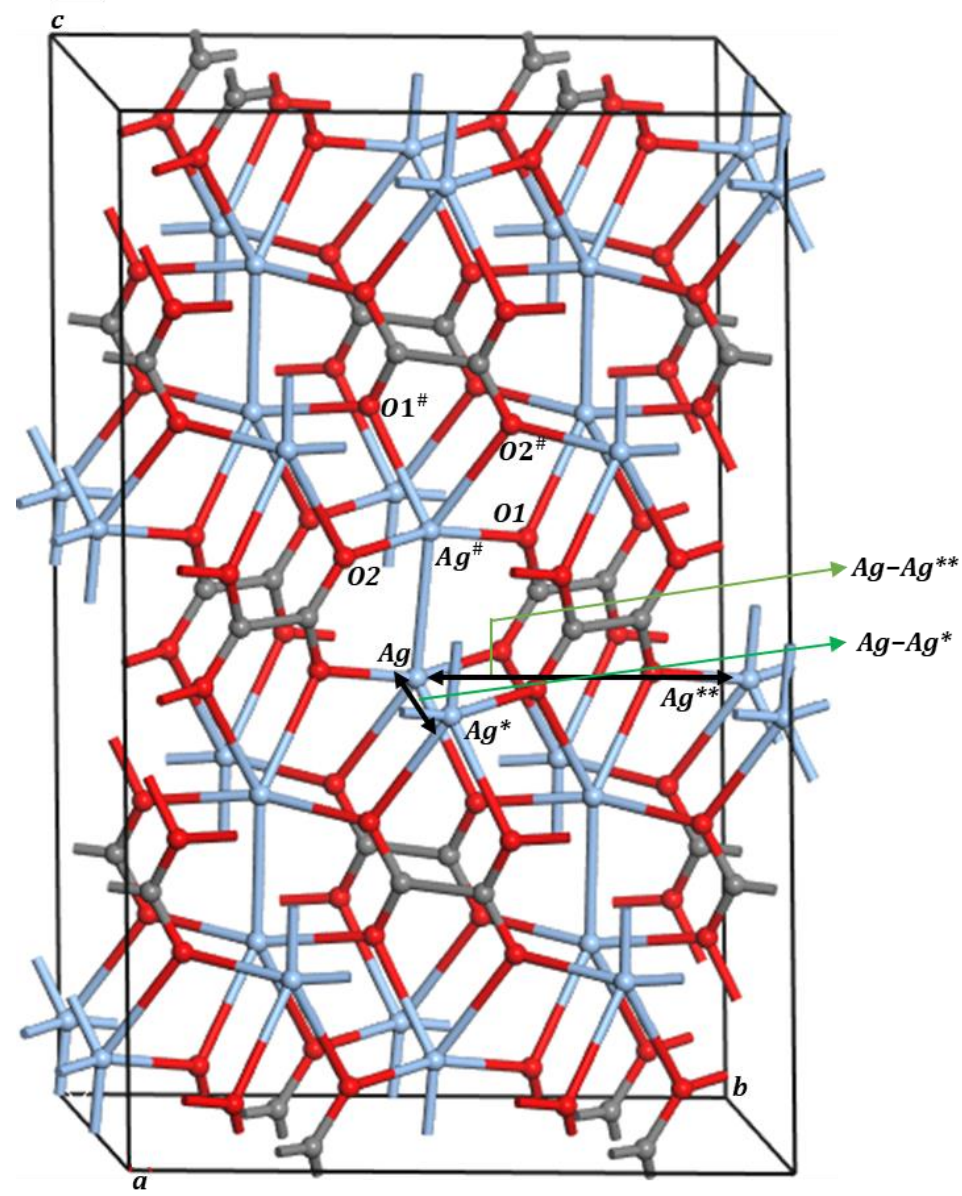




\section{WILEY-VCH}

Figure 3. Elastic properties of silver oxalate as a function of the orientation of the applied strain: (A) Compressibility; (B) Young modulus; (C) Maximum shear modulus; (D) Surfaces of minimum (red) and maximum (blue) Poisson's ratio. The maximum values of the compressibility, Young modulus, shear modulus and Poisson's ratio are $171.36 \mathrm{TPa}^{-1}, 134.04$ $\mathrm{GPa}, 51.29 \mathrm{GPa}$ and 2.58, respectively.
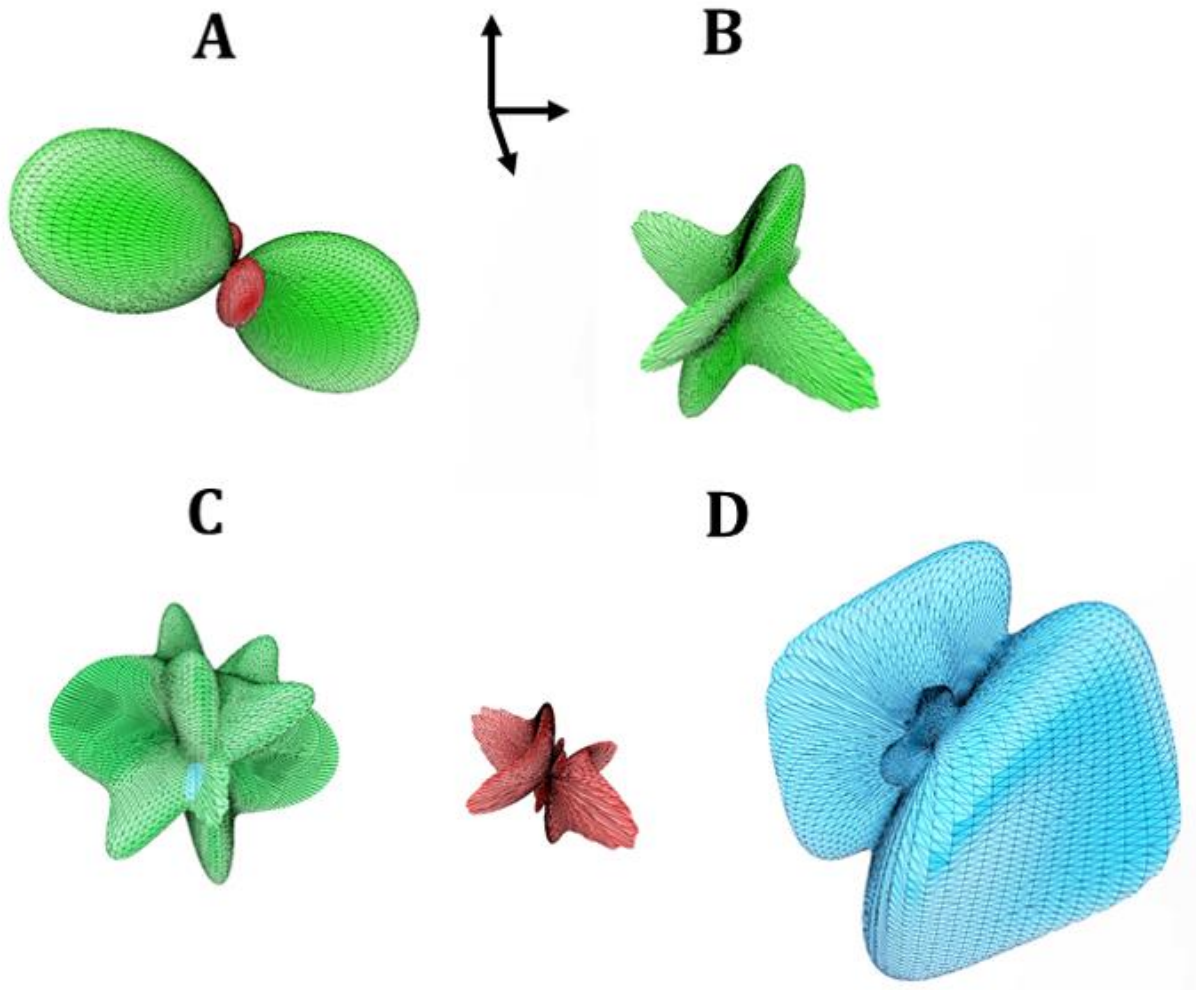

Figure 4. Calculated unit cell volumes and lattice parameters of silver oxalate as a function of the applied external pressure directed along the minimum compressibility direction ( $b$ axis).
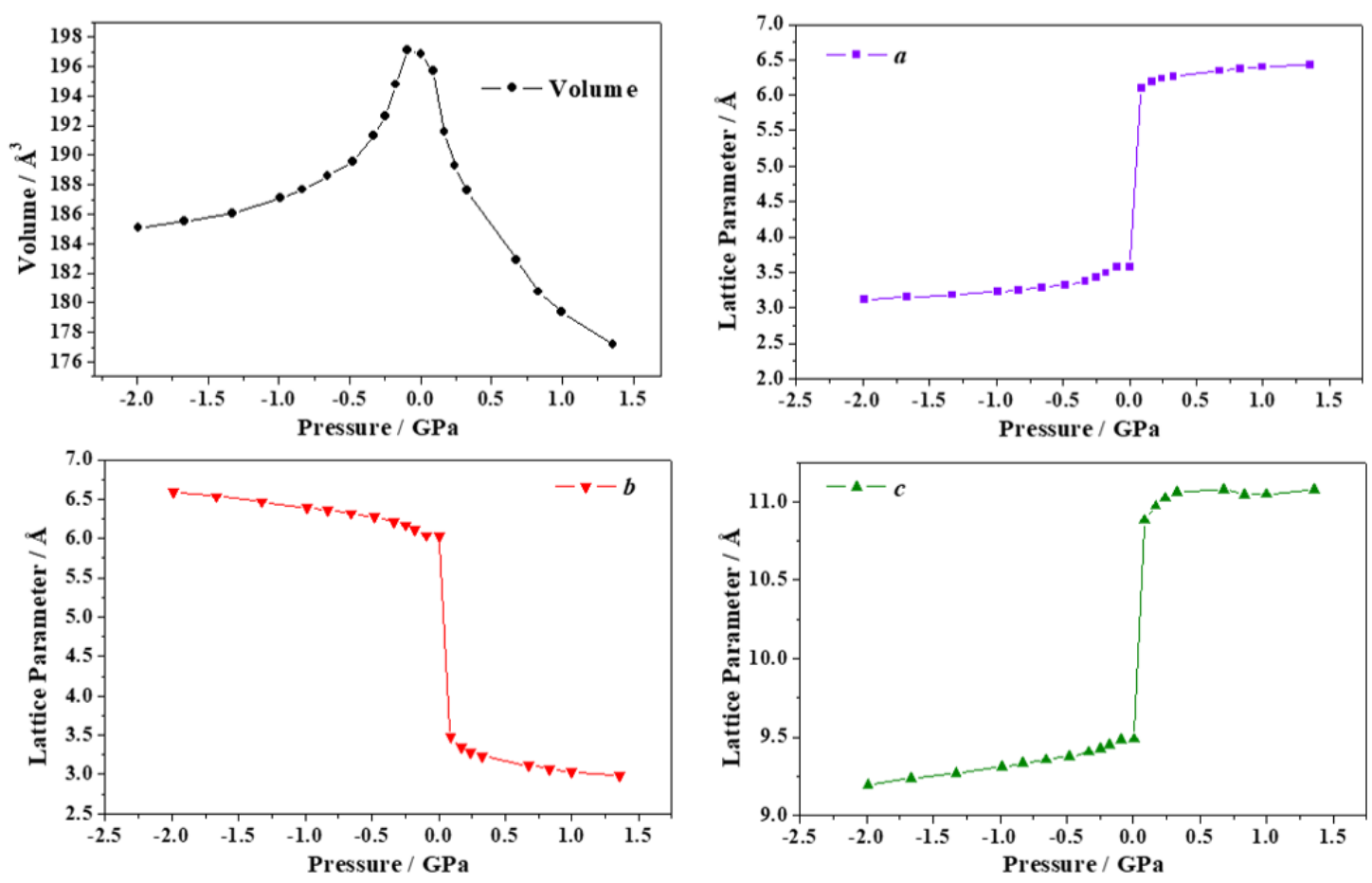


\section{WILEY-VCH}

Figure 5. Calculated compressibilities $\left(k_{V}=-1 / V \cdot(\partial V / \partial P)_{P}\right)$ of silver oxalate as a function of the applied external pressure directed along the minimum compressibility direction ( $b$ axis) in the pressure range from $-2.0 \mathrm{GPa}$ to $0.0 \mathrm{GPa}$.

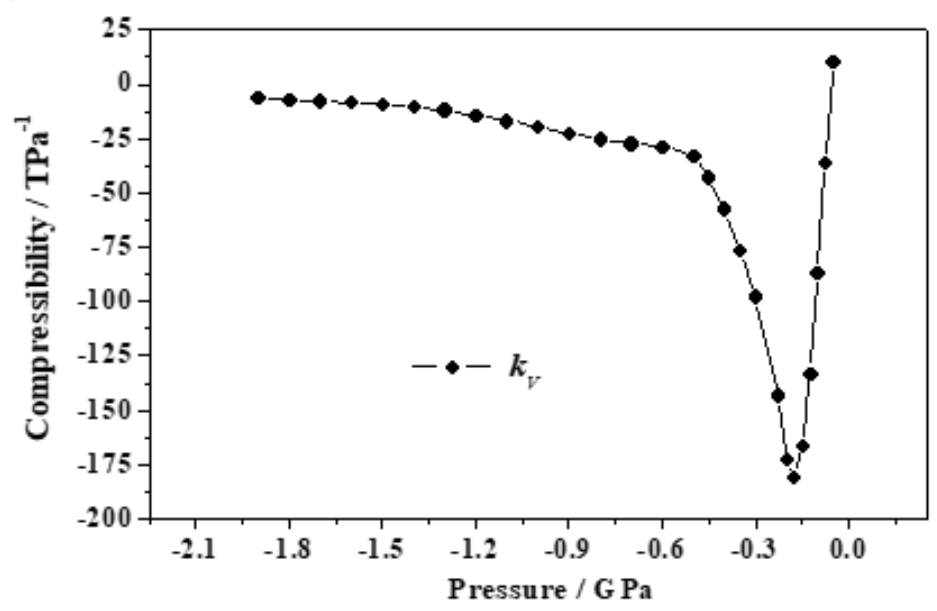

Figure 6. Calculated unit cell volumes and lattice parameters of silver oxalate as a function of the applied isotropic external pressure.
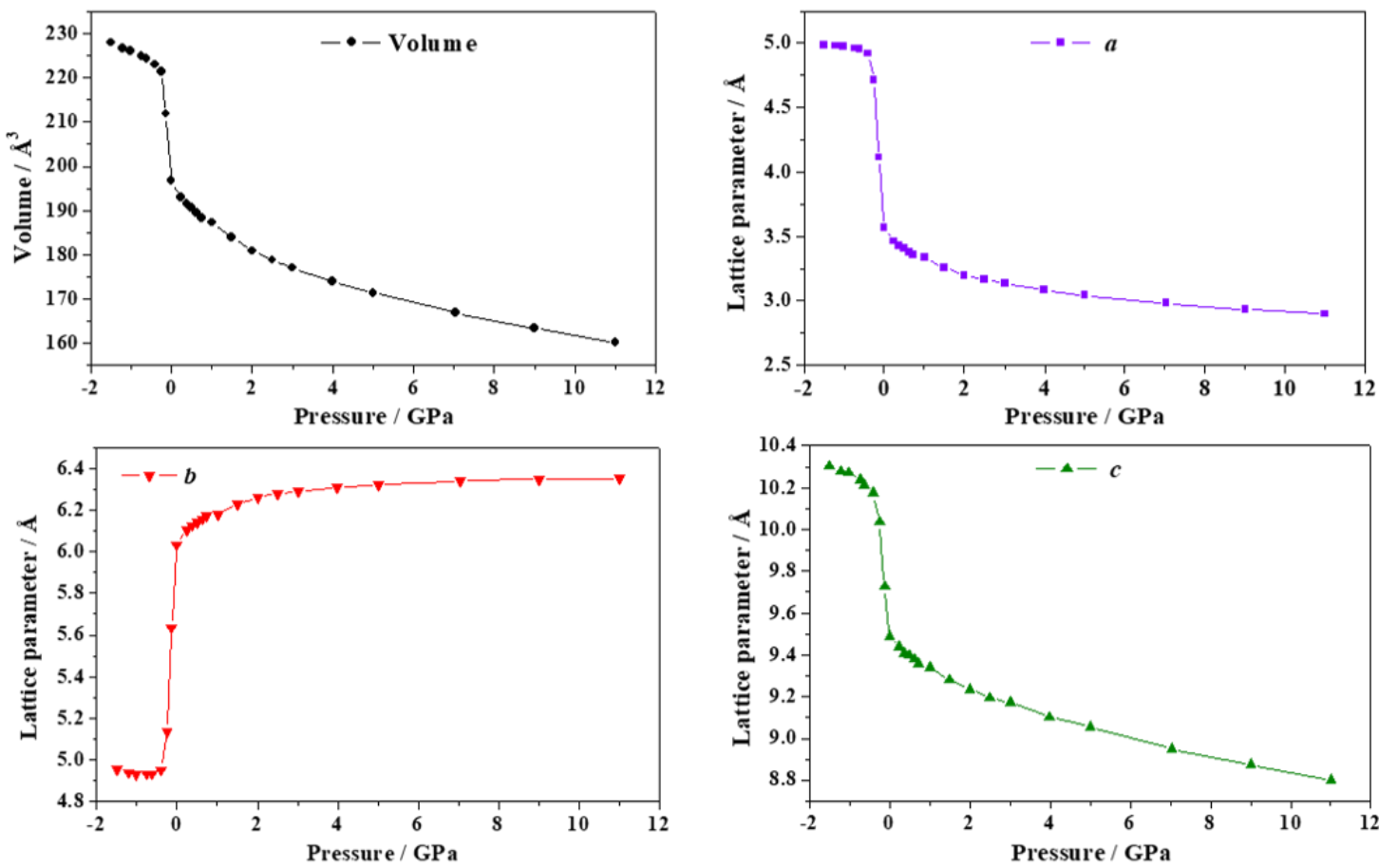


\section{WILEY-VCH}

Figure 7. Calculated compressibilities $\left(k_{b}=-1 / b \cdot(\partial b / \partial P)_{P}\right)$ along $b$ direction of silver oxalate as a function of the isotropic applied isotropic external pressure in the pressure range from $-0.45 \mathrm{GPa}$ to $0.45 \mathrm{GPa}$.

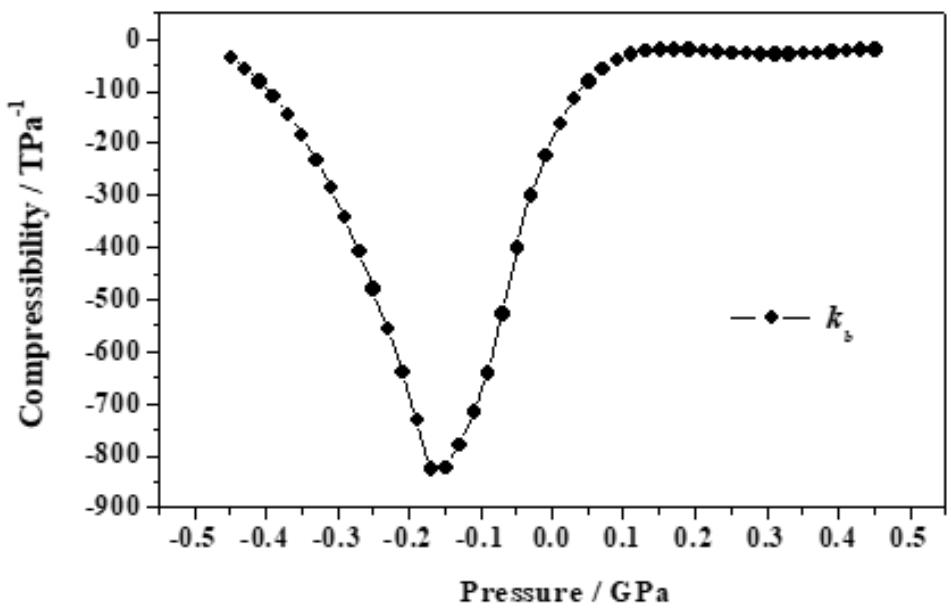

Table 1. Computed mechanical properties of silver oxalate in the Reuss approximation at zero pressure and temperature. The values of the bulk, shear and Young moduli $(B, G$ and $E)$ are given in in GPa. The computed bulk modulus and their pressure derivatives derived from the EOS are also given in the Table.

\begin{tabular}{ccc}
\hline & Property & Value \\
\hline $\boldsymbol{B}$ & Bulk modulus & 9.64 \\
$\boldsymbol{G}$ & Shear modulus & 5.56 \\
$\boldsymbol{E}$ & Young modulus & 13.99 \\
$\boldsymbol{v}$ & Poisson's ratio & 0.26 \\
$\boldsymbol{D}$ & Ductility index & 1.73 \\
$\boldsymbol{H}$ & Hardness index & 0.85 \\
$\boldsymbol{A}^{\boldsymbol{U}}$ & Universal anisotropy index & 26.64 \\
\hline \multicolumn{3}{c}{ EOS } \\
\hline $\boldsymbol{B}$ & Bulk modulus & $12.90 \pm 0.38$ \\
$\boldsymbol{B}^{\prime}$ & Bulk modulus first derivative & $16.84 \pm 0.58$ \\
$\boldsymbol{B}^{\prime \prime}$ & Bulk modulus second derivative & $-10.68 \pm 1.54$ \\
\hline
\end{tabular}

